\title{
Assessment of Right Atrial Function in Patients with First Inferior Myocardial Infarction with \& Without Right Ventricular Involvement by 2D Speckle Tracking Echocardiography
}

\author{
Mohamed Fahmy El-Noamany ${ }^{1}$, Ghada Mahmoud Soltan ${ }^{1}$, Naglaa Fahim Ahmed ${ }^{1}$, \\ Haitham Mohamed Omar El-Ahwal ${ }^{2}$ \\ ${ }^{1}$ Cardiology Department, Faculty of Medicine, Menoufiya University, Shibin El Koum, Egypt \\ ${ }^{2}$ Cardiology Department, Teaching Institutes Organization, Shibin El Koum, Egypt
}

Email address:

Soltanghada@yahoo.com (G. M. Soltan),Dr_Haitham_el_Ahwal@yahoo.com (H. M. O. El-Ahwal)

To cite this article:

Mohamed Fahmy El-Noamany, Ghada Mahmoud Soltan, Naglaa Fahim Ahmed, Haitham Mohamed Omar El-Ahwal. Assessment of Right Atrial Function in Patients with First Inferior Myocardial Infarction with \& Without Right Ventricular Involvement by 2D Speckle Tracking Echocardiography. Cardiology and Cardiovascular Research. Vol. 4, No. 3, 2020, pp. 111-118. doi: 10.11648/j.ccr.20200403.16

Received: May 23, 2020; Accepted: June 18, 2020; Published: July 23, 2020

\begin{abstract}
Background: The right atrium (RA), has received the least attention by researchers. Many reports demonstrated that, RA function may be impaired in the early stages of cardiac disease. The RA plays an important role in maintaining right ventricular (RV) output. RA functional changes have been evaluated in patients with coronary artery disease, but RA affection is not fully elucidated in patients with myocardial infarction (MI) Objective: To assess RA function by 2-Dimensional speckle tracking echocardiography (2D-STE) imaging in patients with acute first left ventricular (LV) inferior wall MI with and without RV involvement. Patients \& methods:-Sixty patients with acute first inferior MI were included in this study; 30 patients had ECG signs of inferior MI without RV infarction (group II) and 30 patients had ECG signs of inferior MI with RV involvement (group III). Twenty five age and sex -matched healthy volunteers were included as a control group (group I). Assessment of RA function was done in all subjects using (2D-STE) for measurements of RA septal and free walls longitudinal strain and strain rate (SR). Results:-RA septal wall \& global systolic strain were significantly reduced in group III (22.5 $\pm 14.8 \%$ and $40.4 \pm 24.5 \%)$ compared to group I $(37.5 \pm 16.3 \%$ and $60.4 \pm 22.4 \%)$ and group II $(35.7 \pm 17.4 \%$ and $54.9 \pm 25.73 \%),(\mathrm{P}<0.001)$, $(\mathrm{P}=0.008)$ respectively. RA septal wall and global early diastolic strain rate were also significantly reduced in group III ($0.77 \pm 0.46 \mathrm{~s}-1$ and $-3.61 \pm 0.55 \mathrm{~s}-1)$ compared to group I (-1.38 $\pm 0.74 \mathrm{~s}-1$ and $-4.41 \pm 0.67 \mathrm{~s}-1)$ and group II $(-1.07 \pm 0.76 \mathrm{~s}-1$ and $-3.73 \pm 0.76 \mathrm{~s}-1), \quad(\mathrm{P}=0.005),(\mathrm{P}<0.001)$ respectively. Conclusion:-In patients with first LV inferior wall MI with $\mathrm{RV}$ involvement, RA functional parameters (RA septal and global strain and SR) were significantly impaired in comparison to healthy controls and patients with first LV inferior wall MI without RV affection. This result may illustrate the significant relation between RV \& RA which is still under research.
\end{abstract}

Keywords: Right Atrium, Inferior MI, Strain, Strain Rate

\section{Introduction}

Myocardial infarction (MI) is a major cause of death and disability worldwide. Coronary atherosclerosis is achronic disease with stable and unstable periods. During unstable periods with activated inflammation in the vascular wall, patients may develop MI. MI may be a minor event in a lifelong chronic disease; it may even go undetected, but it may also be a major catastrophic event leading to sudden death or severe hemodynamic deterioration. A MI may be the first manifestation of coronary artery disease, or it may occur repeatedly in patients with established disease [2].

Right ventricular (RV) infarction may occur alone or in association with left ventricular (LV) inferior wall infarction.

ST-segment elevation in the right precordial lead, V4 R$\mathrm{V} 6 \mathrm{R}$, is one of the most reliable ECG signs of acute RV infarction [3].

The right atrium (RA), has received the least attention by researchers. It has been demonstrated that RA function can be impaired in the early stages of cardiac disease [1]. 
The RA has three functions: reservoir, conduit, and contraction. Reservoir function is the reserving of blood at the time of right ventricular (RV) contraction, conduit function is the direct transferring of blood from the veins to the $\mathrm{RV}$, and contraction function is the booster pumping of blood in late ventricular diastolic time [4].

Accordingly, the RA plays a significant role in maintaining RV output. RA function changes have been evaluated in patients with coronary artery disease, but RA function in patients with myocardial infarction (MI) has yet to be fully elucidated. It has been demonstrated that left atrial (LA) function can be affected by left ventricular myocardial infarction (LVMI), so it stands to reason that RA function may be affected by inferior myocardial infarction. [5, 6]

The RA interacts with the RV with altered RV systolic and diastolic function with an increased filling pressure. It can, therefore, be anticipated that some changes may occur in RA function. [10]

Strain is a measure of tissue deformation. As the ventricle contracts, muscle shortens in the longitudinal and circumferential dimensions (a negative strain) and thickens in the radial direction (a positive strain) [8].

Strain rate (SR) imaging allows the determination of velocity gradients between two points in space. The resulting contraction variable is independent of passive tethering effects from other regions, and therefore appears promising for quantification of regional myocardial function. [7]

Strain \& strain rate techniques are used to assess myocardial movement and deformation. These methods have been frequently used to assess LV function; however, they have yet rarely been used to examine RA function [9], despite RA function is an important prognostic factor in patients with acute first inferior MI [3].

\section{Patients and Methods}

\subsection{Study Population}

The study excluded patients with inferior and anterior MI, previous STEMI, previous coronary artery bypass graft surgery, The presence of a pacemaker or defibrillator lead in the RV, atrial fibrillation, complete right or left bundle branch block, chronic obstructive pulmonary disease, pulmonary hypertension, pulmonary thromboembolic disease, signs of valvular heart disease, organic tricuspid or pulmonary valve disease, dilated cardiomyopathy, myocardial disease, and RV involving, for example, HOCM, amyloidosis, renal dysfunction, pregnancy, severe hypotension (systolic blood pressure $<80 \mathrm{mmHg}$ ), pericardial disease and poor image quality. A total of 60 patients with first inferior MI with and without RVMI were included, in addition to 25 age-matched and sex-matched healthy volunteers as a control group.

A standard 12-lead ECG and a right precordial ECG (lead V4 R) were recorded immediately after arrival to the coronary care unit.

According to MI definition, 30 patients had ECG signs of inferior MI without RV infarction (group II), and 30 patients had ECG signs of inferior MI with RV infarction (group III). In all, 25 age-matched and sex-matched healthy volunteers were included as a control group (group I).

\subsection{Echocardiography}

Echocardiography was performed using a Vivid 9 (GE Vingmed, Horten, Norway) equipped with a harmonic M5S variable frequency $(1.7-4 \mathrm{MHz})$ Phased-array transducer.

The conventional echocardiographic measurements were performed according to the recommendations of the American Society of Echocardiography [11]. Right atrial dimension was determined through the four-chamber view from the maximal medial to lateral dimension at endventricular systole (which corresponds to maximal atrial volume).

\subsection{Assessment of RA Strain Rate and Strain}

Border tracking of the RA was manually traced from the digitized 2D video clips recorded during breath holding and with good quality ECG signal which acquired and stored for off-line analysis using XStrain ${ }^{\mathrm{TM}}$ software with a frame rate between 40-80 fps. The "Zoom/ RES" feature on the echocardiographic machine was used to improve the accuracy of atrial measurements. A circular region of interest was traced on the endocardial cavity interface of the apical four chamber view at end.

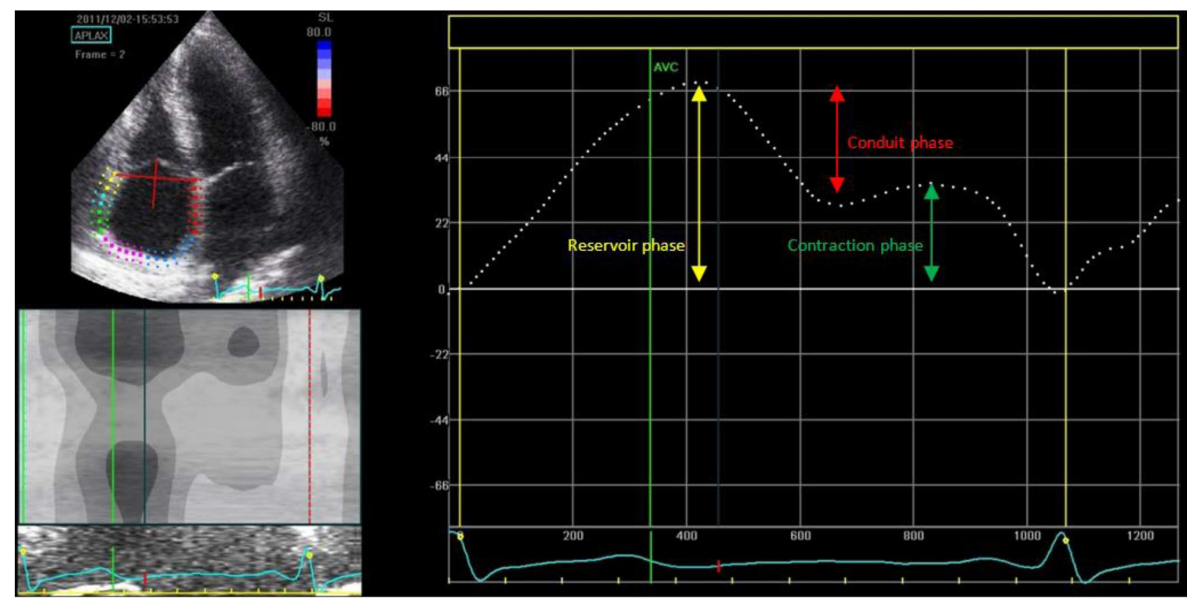

Figure 1. The right atrial strain curve [16]. 

Infarction with \& Without Right Ventricular Involvement by 2D Speckle Tracking Echocardiography

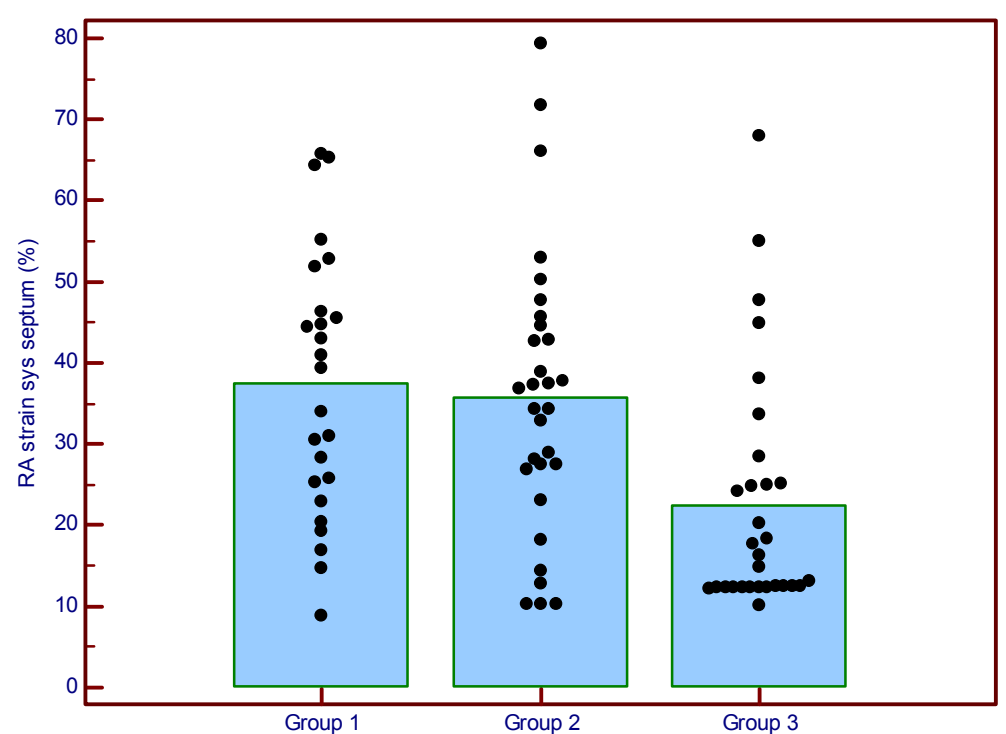

Figure 2. Mean RA strain sys septum in the three study groups. Markers represent individual observations.

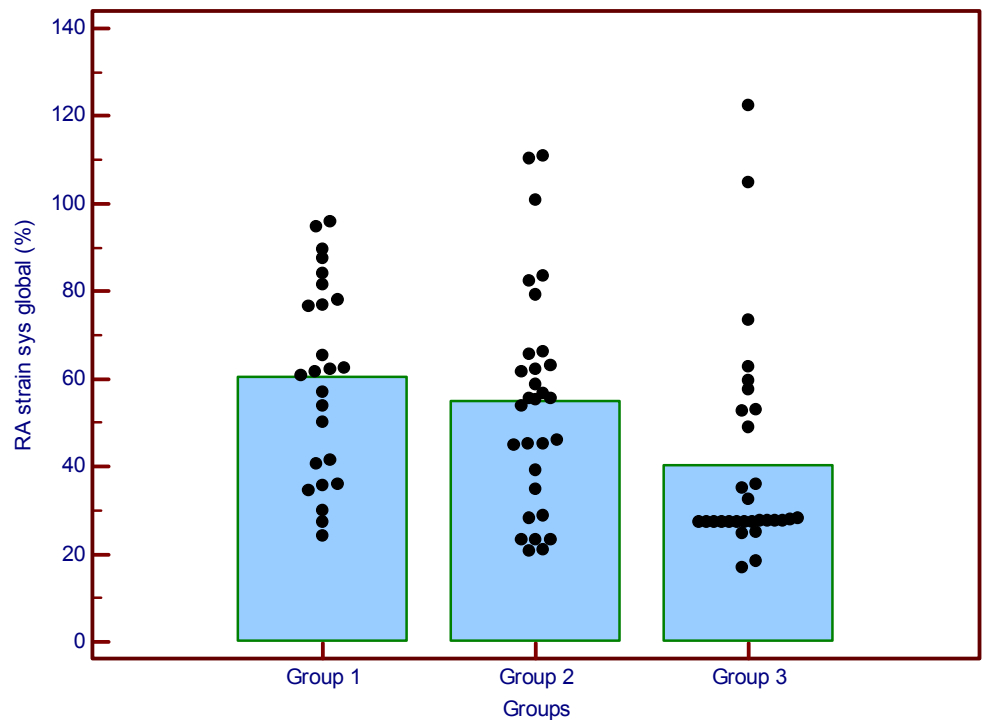

Figure 3. Mean RA strain sys global in the three study groups. Markers represent individual observations.

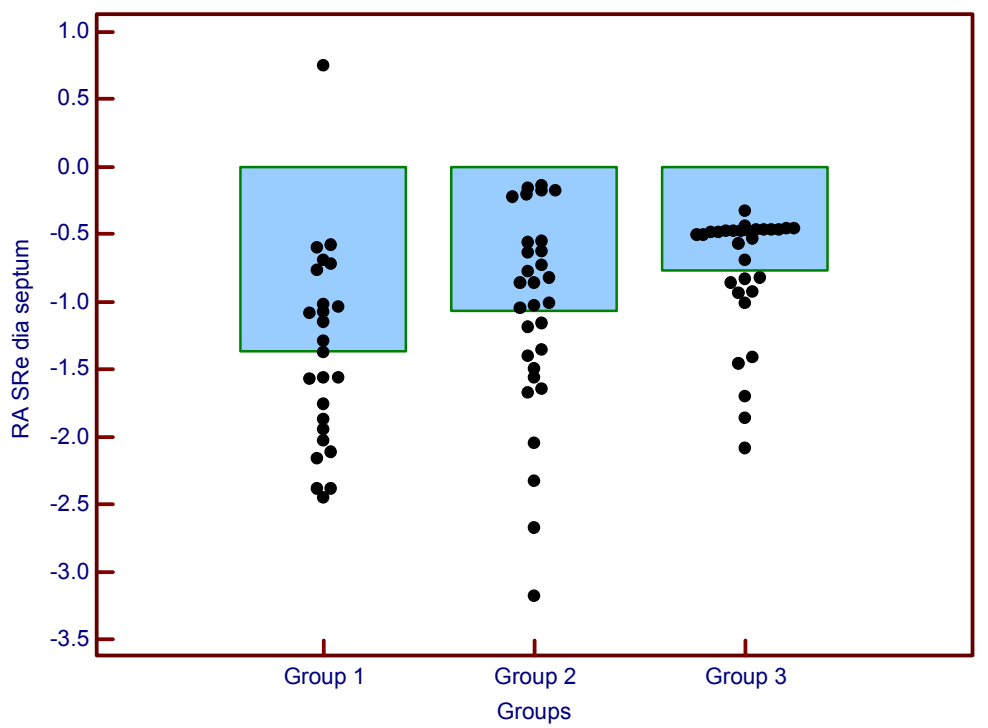

Figure 4. Mean RA SRe dia septum in the three study groups. Markers represent individual observations. 


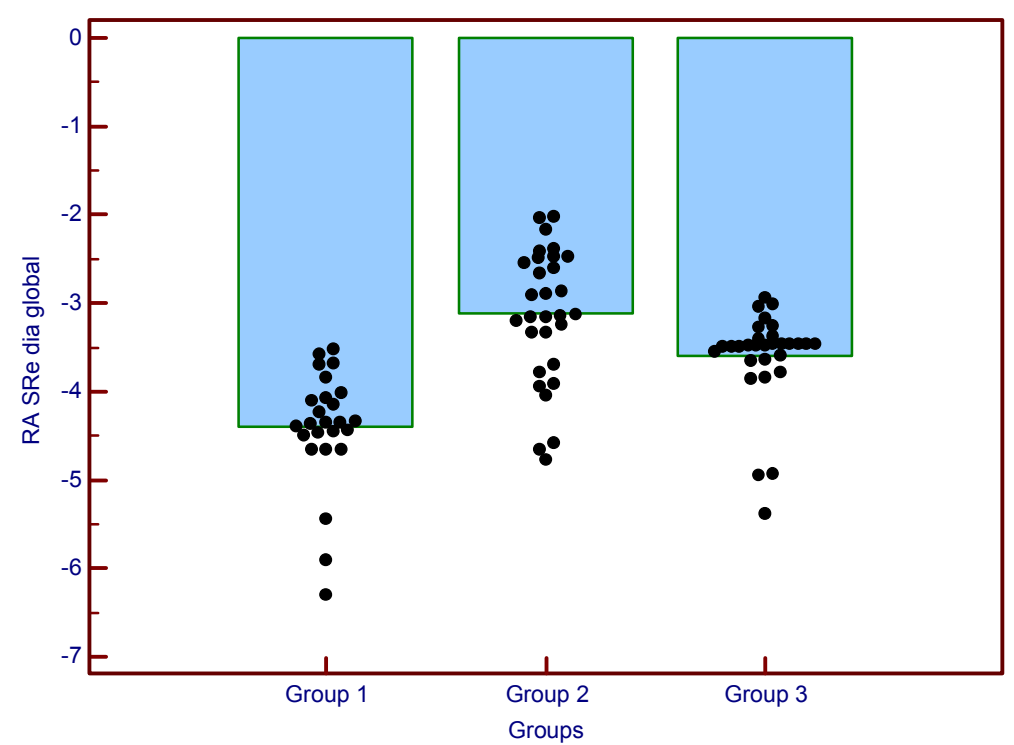

Figure 5. Mean RA SRe dia global in the three study groups. Markers represent individual observations.

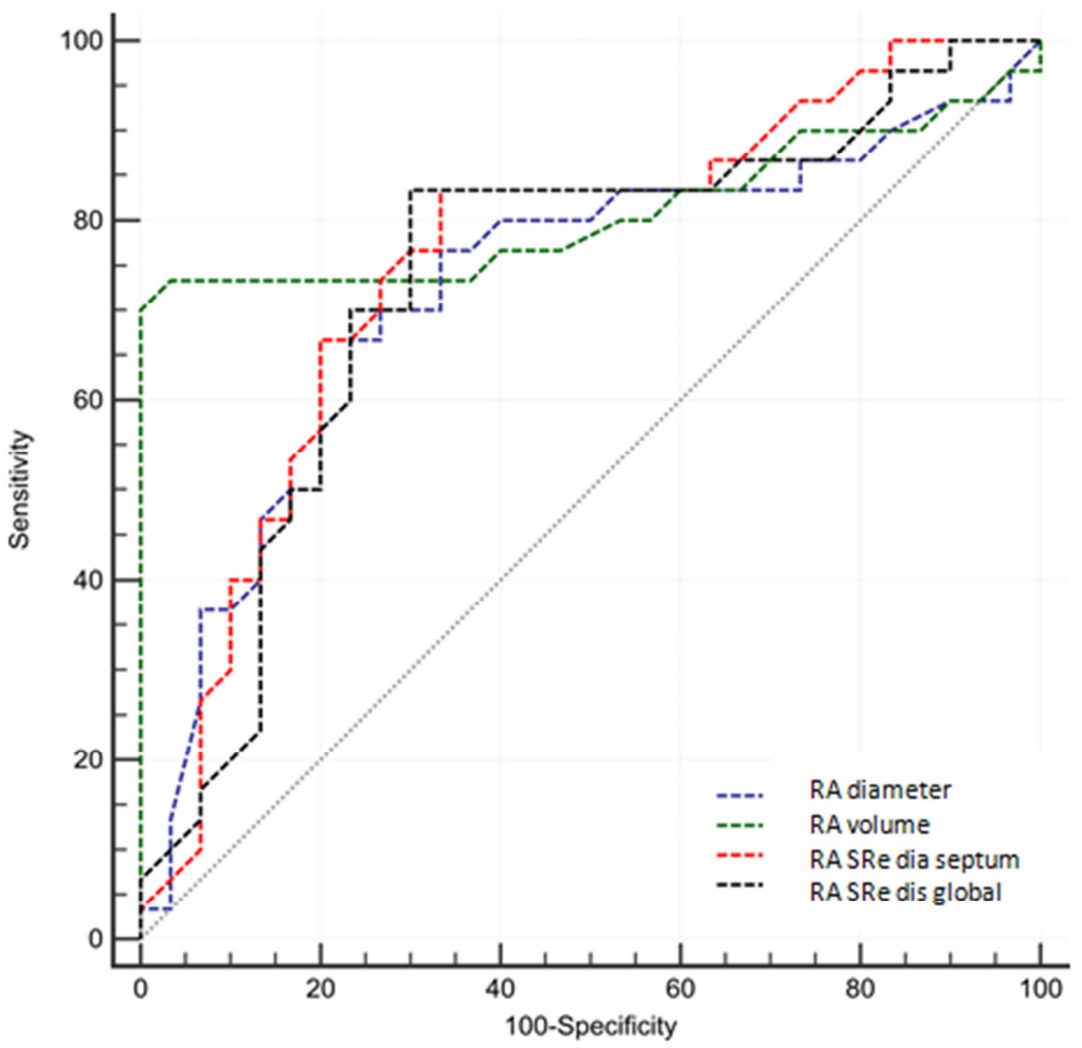

Figure 6. Receiver-operating characteristic (ROC) curves for discrimination between cases of inferior MI with or without RV involvement using RA diameter, $R A$ volume, $R A$ SRe dia septum or $R A$ SRe dia global.

diastole (RA minimum cavity area) using a point-and-click approach. Time-volume curves were extracted from RA wall tracking which provided automatically longitudinal peak velocities achieved by RA walls $1 \mathrm{~cm}$ above the tricuspid annulus in systole, early and late diastole.

Definition of RA endocardial border enabled system to calculate regional longitudinal deformation of the RA walls. Peak systolic strain (Esys) and RA systolic SR (SRsys) were measured as positive curve at LV systole (representing reservoir function), early diastole (SRe) (representing conduit function), atrial diastole (SRa) (representing contractile function).

Image processing algorithm automatically subdivides the atrial wall into 12 segments distributed in septum\& lateral and posterior RA wall-"roof") [12].

\subsection{Statistical Analysis}

Data were analyzed using IBM $\odot$ SPSS $\odot$ Statistics version 
23 (IBM(C) Corp., Armonk, NY) and MedCalc $(\mathrm{C}$ version 18.2.1 (MedCalc $\bigodot$ Software bvba, Ostend, Belgium).

Continuous numerical variables were presented as mean and SD and inter-group differences were compared using one-way analysis of variance (ANOVA) with application of the Tukey-Kramer test for post hoc comparisons if needed.

Categorical variables were presented as number and percentage and differences were compared using the Pearson chi-squared test or Fisher's exact test as appropriate.

Receiver-operating characteristic (ROC) curve analysis was used to examine the value of echocardiographic measures for discrimination between cases of inferior $\mathrm{MI}$ with or without RV involvement. The area under the ROC curve (AUC) is interpreted as follows: table 1

Table 1. Diagnostic value of area under ROC curve (AUC).

\begin{tabular}{ll}
\hline Area under ROC curve (AUC) & Diagnostic value \\
\hline $0.9-1.0$ & Excellent \\
$0.8-0.89$ & Good \\
$0.7-0.79$ & Fair \\
$0.6-0.69$ & Poor \\
$<0.6$ & Fail \\
\hline
\end{tabular}

Two-sided p-values $<0.05$ were considered statistically significant.

Table 2. Comparison of 2D-echocardiographic measures in the three study groups.

\begin{tabular}{|c|c|c|c|c|c|c|c|c|}
\hline \multirow[b]{2}{*}{ Variable } & \multicolumn{2}{|c|}{ Group $1(n=25)$} & \multicolumn{2}{|c|}{ Group $2(n=30)$} & \multicolumn{2}{|c|}{ Group $3(n=30)$} & \multirow{2}{*}{$F(2,82)$} & \multirow{2}{*}{ P-value* } \\
\hline & Mean & SD & Mean & SD & Mean & SD & & \\
\hline LA diameter (mm) & 33.8 & 6.8 & 35.7 & 8.3 & $39.8 \mathrm{a}$ & 9.7 & 3.7057 & $0.029 *$ \\
\hline LA volume (ml) & 26.1 & 3.4 & 29.6 & 3.7 & $39.5 \mathrm{a}, \mathrm{b}$ & 20.9 & 8.277 & $0.001 * *$ \\
\hline LV ESD (mm) & 29.5 & 9.7 & 36.6 & 12.3 & $37.0 \mathrm{a}$ & 12.1 & 3.567 & $0.033 *$ \\
\hline LV EDD (mm) & 43.4 & 9.2 & $50.9 \mathrm{a}$ & 12.0 & 49.9 & 10.7 & 3.807 & $0.026^{*}$ \\
\hline LV FS (\%) & 34.6 & 5.8 & $28.9 \mathrm{a}$ & 8.4 & $28.5 \mathrm{a}$ & 9.0 & 4.912 & $0.010 *$ \\
\hline LV EF (\%) & 63.2 & 8.0 & $54.6 \mathrm{a}$ & 11.8 & $55.2 \mathrm{a}$ & 12.5 & 5.013 & $0.009 * *$ \\
\hline LVPW thickness (mm) & 9.7 & 3.7 & $11.0 \mathrm{a}$ & 3.7 & $11.4 \mathrm{a}$ & 2.7 & 1.785 & 0.174 \\
\hline Mitral E (cm/s) & 0.90 & 0.29 & 0.83 & 0.31 & 0.73 & 0.35 & 2.079 & 0.132 \\
\hline Mitral A $(\mathrm{cm} / \mathrm{s})$ & 0.55 & 0.19 & 0.62 & 0.17 & 0.65 & 0.31 & 1.337 & 0.268 \\
\hline Mitral E/A & 1.59 & 0.74 & 1.38 & 0.47 & $1.21 \mathrm{a}$ & 0.30 & 3.544 & $0.033 *$ \\
\hline Deceleration time (ms) & 179 & 55 & 181 & 55 & 189 & 86 & 0.185 & 0.831 \\
\hline $\mathrm{PAP}(\mathrm{mmHg})$ & 23 & 6 & 26 & 12 & 29 & 17 & 1.120 & 0.331 \\
\hline
\end{tabular}

Data are mean and standard deviation (SD).

$\mathrm{F}=\mathrm{F}$-statistic.

*. One-way analysis of variance (ANOVA).

a. P-value $<0.05$ versus Group 1 (Tukey-Kramer test).

b. P-value $<0.05$ versus Group 2 (Tukey-Kramer test).

\section{Results}

In conventional echocardiography there was highly significant difference was present between group III (Inferior MI with RT MI), group II and group I as regard LA diameter (mm), LA volume (ml) (P-value $<0.001=$ highly significant), LV ESD (mm), LV EDD (mm), LV FS (\%), LV EF (\%),\& Mitral E/A (P-value $<0.05=$ significant). While no significant difference was present between three groups in other data [table 2].

\subsection{Assessment of RA Function}

In conventional data there was no significant difference was present between three groups regarding RA dimensions and RA EF.

\subsection{Assessment of RA Systolic Strain}

A statistically high significant difference was found between group III (Inferior MI with RV MI) versus group I (control) \& group II (Inferior MI without RV MI) regarding septal wall. $(P<0.001)$. A statistically significant difference was found between group III (Inferior MI with RV MI) versus group II Inferior MI without RV MI) regarding RA strain sys global. $(P=0.008)$ [table 3].

Table 3. Comparison of RA strain sys in the three study groups.

\begin{tabular}{|c|c|c|c|c|c|c|c|c|}
\hline \multirow{2}{*}{ Variable } & \multicolumn{2}{|c|}{ Group $1(n=25)$} & \multicolumn{2}{|c|}{ Group $2(n=30)$} & \multicolumn{2}{|c|}{ Group $3(n=30)$} & \multirow{2}{*}{ F $(2,82)$} & \multirow{2}{*}{ P-value* } \\
\hline & Mean & SD & Mean & SD & Mean & SD & & \\
\hline RA strain sys septum (\%) & 37.5 & 16.3 & 35.7 & 17.4 & $22.5 \mathrm{a}, \mathrm{b}$ & 14.8 & 7.408 & $<0.001 * *$ \\
\hline RA strain sys lateral wall (\%) & 86.5 & 34.7 & 80.3 & 43.7 & 63.8 & 42.2 & 2.343 & 0.102 \\
\hline RA strain sys global (\%) & 60.4 & 22.4 & 54.9 & 25.3 & $40.4 \mathrm{~b}$ & 24.5 & 5.147 & $0.008 * *$ \\
\hline
\end{tabular}

Data are mean and standard deviation (SD).

$\mathrm{F}=\mathrm{F}$-statistic.

*. One-way analysis of variance (ANOVA).

a. P-value $<0.05$ versus Group 1 (Tukey-Kramer test).

b. P-value $<0.05$ versus Group 2 (Tukey-Kramer test). 


\subsection{Assessment of RA SRe Dia \& SRa Dia}

A statistically high significant difference was found between group III (Inferior MI with RT MI) versus group II (Inferior MI without RT MI) regarding RA. SRe dia septum \& global. $(P=0.005)$, $(P<0.001)$ respectively [table4].

No significant difference was present between three groups regarding RA SRa dia.

Table 4. Comparison of RA SRe dia in the three study groups.

\begin{tabular}{|c|c|c|c|c|c|c|c|c|}
\hline \multirow{2}{*}{ Variable } & \multicolumn{2}{|c|}{ Group $1(n=25)$} & \multicolumn{2}{|c|}{ Group $2(n=30)$} & \multicolumn{2}{|c|}{ Group $3(n=30)$} & \multirow{2}{*}{$F(2,82)$} & \multirow{2}{*}{ P-value* } \\
\hline & Mean & SD & Mean & SD & Mean & SD & & \\
\hline RA SRe dia septum & -1.38 & 0.74 & -1.07 & 0.76 & $-0.77 b$ & 0.46 & 5.659 & $0.005 * *$ \\
\hline RA SRe dia lateral wall & -2.37 & 0.92 & -1.98 & 0.97 & -1.85 & 0.84 & 2.353 & 0.101 \\
\hline RA SRe dia global (\%) & -4.41 & 0.67 & -3.13 & 0.76 & $-3.61 b$ & 0.55 & 25.454 & $0.000 * *$ \\
\hline
\end{tabular}

Data are mean and standard deviation (SD).

$\mathrm{F}=\mathrm{F}$-statistic.

*. One-way analysis of variance (ANOVA).

b. P-value $<0.05$ versus Group 2 (Tukey-Kramer test).

* sighificance.

** highly sighificance.

\section{Discussion}

A few studies showed that strain and strain rate imaging appears good for functional assessment of the right atrium. the accuracy of strain rate imaging to quantify RA deformation that can be occur as a complication of inferior STEMI specialy if associated with RV affection.

In our study 60 patients with acute inferior MI were examined for RA strain \& strain rate 30 patients had inferior MI without RV infarction (group II) and 30 patients had inferior MI with RV infarction (group III). In all, 25 agematched and sex-matched healthy volunteers were included as a control group (group I).

Statistically high significant difference was found between group III (Inferior MI with RV MI) versus group I (control)
\& group II (Inferior MI without RV MI) regarding septal wall systolic strain \& RA global systolic strain. $(P<0.001)$, $(P=0.008)$ [table 3].

statistically high significant difference was found between group III (Inferior MI with RT MI) versus group II (Inferior MI without RT MI) regarding RA.

SRe dia septum \& global. $(P=0.005), \quad(P<0.001)$ respectively [table 4].

In Nourian et al study that assessed RA reservoir, conduit $\&$ contractile functions in 70 patients with inferior MI (43 patients without RVMI \& 27 patients with RVMI) shows that the 2D speckle tracking echocardiography (2DSTE) for the evaluation of RA function showed that RA SRe, and RA SRs were lower in the patients with INFMI + RVMI than in the patients.

Table 5. Receiver-operating characteristic (ROC) curve analysis for discrimination between cases of inferior MI with or without RV involvement using RA diameter, RA volume, RA SRe dia septum or RA SRe dia global.

\begin{tabular}{|c|c|c|c|c|}
\hline & Predictor & & & \\
\hline ROC metric & RA diameter & RA volume & RA SRe dia septum & RA SRe dia global \\
\hline AUC & 0.723 & 0.809 & 0.757 & 0.735 \\
\hline SE & 0.069 & 0.063 & 0.065 & 0.068 \\
\hline $95 \% \mathrm{CI}$ & 0.592 to 0.831 & 0.687 to 0.899 & 0.629 to 0.859 & 0.605 to 0.841 \\
\hline Z statistic & 3.238 & 4.879 & 3.985 & 3.460 \\
\hline $\mathrm{P}$-value $(\mathrm{AUC}=0.5)$ & $0.001 * *$ & $<0.0001 * *$ & $<0.001 * *$ & $0.001 * *$ \\
\hline Youden index $(\mathrm{J})$ & 0.433 & 0.700 & 0.500 & 0.533 \\
\hline Sensitivity (\%) & 76.7 & 73.3 & 83.3 & 83.3 \\
\hline $95 \% \mathrm{CI}$ & $57.7-90.1$ & $54.1-87.7$ & $65.3-94.4$ & $65.3-94.4$ \\
\hline Specificity (\%) & 66.7 & 96.7 & 66.7 & 70.0 \\
\hline $95 \% \mathrm{CI}$ & $47.2-82.7$ & $82.8-99.9$ & $47.2-82.7$ & $50.6-85.3$ \\
\hline$+\mathrm{LR}$ & 2.3 & 22.0 & 2.5 & 2.8 \\
\hline $95 \% \mathrm{CI}$ & $1.3-4.0$ & $3.2-153.0$ & $1.5-4.3$ & $1.6-4.9$ \\
\hline -LR & 0.4 & 0.3 & 0.3 & 0.2 \\
\hline $95 \% \mathrm{CI}$ & $0.2-0.7$ & $0.2-0.5$ & $0.1-0.6$ & $0.1-0.5$ \\
\hline $95 \% \mathrm{CI}$ & $57.2-79.8$ & $76.0-99.4$ & $59.5-81.0$ & $61.1-83.1$ \\
\hline -PV (\%) & 74.1 & 78.4 & 80.0 & 80.8 \\
\hline $95 \% \mathrm{CI}$ & $58.7-85.1$ & $66.6-86.8$ & $63.3-90.3$ & $64.6-90.6$ \\
\hline
\end{tabular}

$\mathrm{AUC}=$ area under ROC curve, $\mathrm{SE}=$ standard error, $95 \% \mathrm{CI}=9 \% \%$ confidence interval, $+\mathrm{LR}=$ positive likelihood ratio, - $\mathrm{LR}=$ negative likelihood ratio, $+\mathrm{PV}=$ positive predictive value, $-\mathrm{PV}=$ negative predictive value. 
III (Inferior MI with RT MI) versus group I (control) \& group II (Inferior MI without RT MI) regarding septal wall \& RA global systolic strain $(\mathrm{P}-$ value $=<0.001,0.008)$ respectively [13].

In our study the area under the curve for RA diameter, RA volume, RA SRe dia septum \& RA SRe dia global for INFMI + RVMI discrimination was $0.723(95 \%$ CI $0.592-0.831$; P value $=0.001), 0.809(95 \%$ CI $0.687-0.899 ; \mathrm{P}$ value $<0.0001)$, 0.757 (95\% CI 0.629 - 0.859; P value <0.001), $0.735(95 \%$ CI 0.605 to 0.841 ; P value $=0.001)$, respectively.

RA diameter has $76.7 \%$ Sensitivity \& $66.7 \%$ specificity, RA volume has $73.3 \%$ Sensitivity \& $96.7 \%$ specificity, RA SRe dia septum has $83.3 \%$ Sensitivity \& $66.7 \%$ specificity \& RA SRe dia global has $83.3 \%$ Sensitivity \& $70 \%$ specificity for the discrimination of INFMI + RVMI from INFMI.

Also in Nourian et al. study The area under the curve for EDS, RA SRE, RA SRS, expansion index and diastolic emptying index, for INFMI + RVMI discrimination was $0.682(95 \%$ CI $0.550-0.815 ; \mathrm{p}$ value $=0.011), 0.640(95 \% \mathrm{CI}$ $0.512-0.768 ; \mathrm{p}$ value $=0.040), 0.400(95 \%$ CI $0.269-0.530 ; \mathrm{p}$ value $=0.141), 0.338(95 \%$ CI $0.209-0.467 ; \mathrm{p}$ value $=0.017)$, and $0.338(95 \%$ CI $0.209-0.467 ; \quad \mathrm{p}$ value $=0.017)$, respectively. EDS $<27.5 \%$ had $59.3 \%$ sensitivity and $79.1 \%$ specificity for the discrimination of INFMI + RVMI from INFMI. [13]

Our study demonstrated that right atrial reservoir ( $\&$ sys, SRsys) and conduit (early diastolic SRe) function were significantly reduced in patients with inferior STEMI with RV (group III) versus group II \& control group. [13]

These results are concordant with the results of Badran $e$ al study that studied Right atrium deformation in 118 patients with HCM \& 33 healthy subjects as a control group that shows also marked decrease right atrial reservoir ( $\varepsilon$ sys, SRsys) and conduit (early diastolic SRe) function in patients with HCM versus control group. [14]

Our study showed that right atrial reservoir (\&sys, SRsys) and conduit (early diastolic SRe) function were significantly reduced in patients with inferior STEMI with RV (group III) versus group II \& control group.

This is also concordant with OjaghiHaghighi et al, that studied thirty patients with a diagnosis of heart failure and the control group consisted of 32 healthy adults matched for age and sex. The right atrial deformation indices were significantly compromised in the heart failure patients versus the normal subjects $(P=0.0001)$. [15]

In our study also there was no difference between patients who had inferior MI \& those who had also RV affection regarding RA SRA which is discordant with Yan et al. that evaluated RA function with velocity vector imaging in patients with coronary artery disease without MI and found that RA SRA was increased in those with severe coronary artery disease compared with the ones with normal coronary arteries. [17]

Also while our study shows that there was marked decrease in RA functions in Patients with Inferior with RVMI, Dogan et al. compared LA function between patients with ST-elevation MI treated with primary PCI and healthy subjects in the first $48 \mathrm{~h}$ after hospital admission and measured only 1 single 2DSTE-derived parameter, Systolic strain, they found that systolic strain, as a marker of reservoir function, was reduced in the patients with MI. In addition, diastolic emptying index was reduced in the patient group. [5]

However, LA volumes (maximal LA volume, minimal LA volume, and pre-A LA volume) were larger in the patient group than in the normal subjects. [5]

Additionally, diastolic emptying index and passive emptying index were reduced. The authors also reported that LA volumes were higher in the patients with non-STsegment elevation MI than in the control subjects.

Also antoni et al get concordant with our results but regarding LA functions when studied LA strain in 320 patients with acute MI treated with primary PCI within 48 hours compared with 35 normal subjects. There was significant decrease in LA reservoir function $(140 \pm 65$ vs $164 \pm 41 \%,(p=0.03) \&$ lower LA strain $39 \pm 10 v s 33 \pm 11 \%$, $(\mathrm{p}=0.002)$ in comparison with normal subjects. [16]

The same also in Magdy et al study, 40 patients presented with acute STEMI compared with 20 healthy subjects as a control group regarding LA strain \& strain rate. All the LA global longitudinal strain $(\%)$ and strain rate $\left(\mathrm{S}^{-1}\right)$ parameters were significantly reduced in patient group compared to control group, the $\varepsilon$ sys $(p=0.0001)$, the SRsys $(p=0.0001)$, the SRe $(p=0.0001)$, and the SRa $(p=0.034)$ [18].

Our findings may illustrate the significance of the evaluation of RA function because it permits us to evaluate $\mathrm{RV}$ function from another window. According to the results of our study, some 2DSTE-derived indices of RA function were sensitive to RV dysfunction in the presence of RVMI because the appearance of impaired RA function (as evaluated by 2DSTE) preceded the appearance of the difference in the 2DSTE-derived indices of RV function.

We found impaired RA reservoir and conduit functions and preserved RA contraction function in our patients with INFMI + RVMI.

\section{Limitations}

Speckle tracking method is influenced by image quality, and in particular clinical conditions, they can present limitations due to the physiological growth of the myocardial chambers, which prevents, sometimes, the perfect framing of the image in the echocardiographic window. The resolution of $2 \mathrm{D}$ imaging may be a problem in some subjects, and inadequate border recognition of the right atrium may be another factor limiting assessment of myocardial strain. Myocardial contractility is a complex mechanism; in the present study, we investigated only the longitudinal deformation while the other components of wall deformation, like radial strain, circumferential are not included. However, approaching myocardial function using speckle tracking; is non-angle dependent and more reproducible modality that better reflects myocardial function and may allow easier patient follow up in our daily practice? 


\section{Conclusion}

In patients with first LV inferior wall MI with RV involvement, RA functional parameters (RA septal and global strain and SR) were significantly impaired in comparison to healthy controls and patients with first LV inferior wall MI without RV affection. This result may illustrate the significant relation between RV \& RA which is still under research.

\section{References}

[1] Tadic M. (2015) The right atrium, a forgotten cardiac chamber: an updated review of multimodality imaging. J Clin Ultrasound.

[2] Kristian T, JS Alpert, Harvey D, White (2007) HDJoint ESC/ACCF/AHA/WHF Task Force for the Redefinition of Myocardial Infarction. Universal definition of myocardial infarction. Eur Heart J.

[3] Braat SH, Brugada P, de Zwaan C, Coenegracht JM, Wellens HJ. (1983) Value of electrocardiogramin detecting right ventricular involvement in patients with acute inferior wall myocardial infarction. Heart J.

[4] D'Alto M, Scognamiglio G, Dimopoulos K, Bossone E, Vizza D, Romeo E, Vonk-Noordergraaf A, Gaine S, Peacock A, Naeije R (2015) Right heart and pulmonary vessels structure and function.

[5] Dogan C, Ozdemir N, Hatipoglu S, Bakal RB, Omaygenc MO, Dindar B, Candan O, Emiroglu MY, Kaymaz C (2013) Relation of left atrial peak systolic strain with left ventricular diastolic dysfunction and brain natriuretic peptide level in patients presenting with ST-elevation myocardial infarction.

[6] Jing Z, Jianchang C, Weiting X, Lan G, Shaikh F, Yanni W (2013) Comparison of left atrial function in healthy individuals versus patients with non-ST-segment elevation myocardial infarction using two-dimensional speckle tracking echocardiography. Cardiovase J Afr.

[7] Elnoamany M, Ahmed N, Ragab E (2014) Echocardiographic assessment of right ventricular function in patients with pulmonary hypertension: strain imaging study. Menoufia Med J.

[8] Monaster S, Ahmad M, Braik A (2014). Comparison between strain and strain rate in hypertensive patients with and without left ventricular hypertrophy: a speckle-tracking study. Menoufia Med J.

[9] Chockalingam A, Gnanavelu G, Alagesan R, Subramaniam T.
(2004) Myocardial performance index in evaluation of acute right ventricular myocardial infarction. Echocardiography.

[10] Goldstein JA (2012) Acute right ventricular infarction. Cardiol Clin.

[11] Schiller, N. B., Shah, P. M., Crawford, M., DeMaria, A., Devereux, R., Feigenbaum, H.,... Tajik, A. J. (1989). Recommendations for Quantitation of the Left Ventricle by Two-Dimensional Echocardiography. Journal of the American Society of Echocardiography, 2 (5), 358-367. doi: 10.1016/s0894-7317(89)80014-8.

[12] Bussadori, C., Moreo, A., Di Donato, M., De Chiara, B., Negura, D., Dall'Aglio, E., Carminati, M. (2009). A new 2Dbased method for myocardial velocity strain and strain rate quantification in a normal adult and paediatric population: assessment of reference values. Cardiovascular Ultrasound, 7 (1). doi: $10.1186 / 1476-7120-7-8$.

[13] Nourian, S., Hosseinsabet, A., Jalali, A., \& MohseniBadalabadi, R. (2016). Evaluation of right atrial function by two-dimensional speckle-tracking echocardiography in patients with right ventricular myocardial infarction. The International Journal of Cardiovascular Imaging, 33 (1), 47 56.

[14] Badran HM, Soltan G, Hassan H, Nazmy A, Faheem N, Saadan H, Yacoub MH. (2012) Changes in left atrial deformation in hypertrophic cardiomyopathy: Evaluation by vector velocity imaging, Global Cardiology Science \& Practice.: 25 .

[15] Haghighi, Z. O., Naderi, N., Amin, A., Taghavi, S., Sadeghi, M., Moladoust, H.,... Haghighi, H. O. (2011). Quantitative assessment of right atrial function by strain and strain rate imaging in patients with heart failure. Acta Cardiologica, 66 (6), 737-742. doi: 10.1080/ac.66.6.2136957.

[16] Antoni, M. L., ten Brinke, E. A., Atary, J. Z., Marsan, N. A., Holman, E. R., Schalij, M. J., Delgado, V. (2011). Left atrial strain is related to adverse events in patients after acute myocardial infarction treated with primary percutaneous coronary intervention. Heart, 97 (16), 1332-1337. 2011.

[17] Yan P, Sun B, Shi H, Zhu W, Zhou Q, Jiang Y, Zhu H, Huang $\mathrm{G}$ (2012). Left atrial and right atrial deformation in patients with coronary artery disease: a velocity vector imaging-based study. PLoS One 7: e51204.

[18] Magdy, G., El Ashmawy, H., Zidan, A., \& Saeed, A. (2016). Left atrial myocardial deformation characteristics in patients presenting with ST elevation myocardial infarction. The Egyptian Heart Journal, 68 (3), 181-186. doi: 10.1016/j.ehj.05.003. 